\title{
ANÁLISE EPIDEMIOLÓGICA DA COINFECÇÃO TUBERCULOSE/HIV
}

\author{
Layze Braz de Oliveira ${ }^{1}$, Christefany Régia Braz Costar ${ }^{1}$, Artur Acelino Francisco Luz Nunes Queiroz ${ }^{2}$, Telma \\ Maria Evangelista de Araújo ${ }^{3}$, Karinna Alves Amorim de Sousa ${ }^{4}$, Renata Karina Reis ${ }^{5}$
}

RESUMO: Objetivo: analisar o perfil epidemiológico da coinfecção tuberculose e o vírus da imunodeficiência. Método: estudo descritivo, realizado com levantamento na base de dados do Sistema de Informação de Agravos de Notificação no estado do Piauí, no período de 2007 a 2016. A coleta ocorreu por meio de instrumento contendo características sociodemográficas e relacionadas à coinfecção Tuberculose/vírus da imunodeficiência. Realizouse análise descritiva simples. Resultados: A prevalência da coinfecção tuberculose e vírus da imunodeficiência foi de $6,1 \%$ pacientes $(n=619)$. Os dados mostram a predominância de indivíduos do sexo masculino ( $n=467 / 75,4 \%$ ), adultos com faixa etária entre 20 a 49 anos $(n=492 / 79,5 \%$ ) e pardos $(n=457 / 73,8 \%)$. A forma clínica da tuberculose mais encontrada foi a pulmonar com $n=412(66,6 \%)$ casos. O percentual de cura dos destes pacientes foi de 60,8\%. Conclusão: Os resultados apontaram uma alta prevalência dessa coinfecção e uma elevada detecção da infecção do vírus da imunodeficiência em pacientes com tuberculose.

DESCRITORES: Tuberculose; HIV; Prevalência; Epidemiologia; Coinfecção.

\section{EPIDEMIOLOGICAL ANALYSIS OF TUBERCULOSIS/HIV COINFECTION}

\begin{abstract}
Objective: analyze the epidemiological profile of coinfection by tuberculosis and the immunodeficiency virus. Method: descriptive study, developed based on a survey in the database of the Disease Notification System in the State of Piauí between 2007 and 2016. The data were collected through an instrument with sociodemographic characteristics and data related to the coinfection by tuberculosis and the immunodeficiency virus. Simple descriptive analysis was applied. Results: The prevalence of coinfection by tuberculosis and the immunodeficiency virus amounted to $6.1 \%$ of the patients $(n=619)$. The data show the predominance of male individuals $(n=467 / 75.4 \%)$, adults between 20 and 49 years old $(n=492 / 79.5 \%)$ and mulattos $(n=457 / 73.8 \%)$. The most identified clinical form of tuberculosis was the pulmonary with $\mathrm{n}=412(66.6 \%)$ cases. These patients' cure percentage corresponded to $60.8 \%$. Conclusion: The results appointed a high prevalence of this coinfection and a high detection of the infection by the immunodeficiency virus in tuberculosis patients.
\end{abstract}

DESCRIPTORS: Tuberculosis; HIV; Prevalence; Epidemiology; Coinfection.

\section{ANÁLISIS EPIDEMIOLÓGICO DE LA COINFECCIÓN TUBERCULOSIS/VIH}

RESUMEN: Objetivo: analizar el perfil epidemiológico de la coinfección por la tuberculosis y el virus de la inmunodeficiencia. Método: estudio descriptivo con búsqueda en la base de datos del Sistema de Información de Agravios de Notificación en el estado de Piauí, en el período de 2007 a 2016. Los datos fueron recolectados mediante instrumento con características sociodemográficas y relacionadas a la coinfección Tuberculosis/virus de la inmunodeficiencia. Fue aplicado análisis descriptivo simple. Resultados: La prevalencia de la coinfección tuberculosis y virus de la inmunodeficiencia correspondió al 6,1\% de los pacientes ( $\mathrm{n}=619$ ). Los datos muestran la predominancia de individuos del sexo masculino $(n=467 / 75,4 \%)$, adultos con rango de edad entre 20 y 49 años $(n=492 /$ $79,5 \%)$ y pardos $(n=457 / 73,8 \%)$. La forma clínica de la tuberculosis más encontrada fue la pulmonar con $n=412(66,6 \%)$ casos. $E$ I porcentaje de cura de estos pacientes fue del 60,8\%. Conclusión: Los resultados indicaron alta prevalencia de esa coinfección y alta detección de la coinfección por el virus de la inmunodeficiencia en pacientes con tuberculosis.

DESCRIPTORES: Tuberculosis; VIH; Prevalencia; Epidemiología; Coinfección.

${ }^{1}$ Enfermeira. Mestranda em Enfermagem. Universidade de São Paulo. Ribeirão Preto, SP, Brasil.

2Enfermeiro. Mestrando Enfermagem. Universidade de São Paulo. Ribeirão Preto, SP, Brasil.

${ }^{3}$ Enfermeira. Doutora em Enfermagem. Docente da Universidade Federal do Piauí. Teresina, PI, Brasil.

${ }^{4}$ Enfermeira. Especialista em Gestão em programas de controle da Tuberculose. Coordenadora de Doenças transmissíveis da Secretaria de Estado da Saúde do Piauí. Teresina, PI, Brasil.

${ }^{5}$ Enfermeira. Doutora em Enfermagem. Docente de Enfermagem da Universidade de São Paulo. Ribeirão Preto, SP, Brasil.

Autor Correspondente:

Recebido: 05/03/2017

Layze Braz de Oliveira

Finalizado: 04/12/2017

Universidade de São Paulo

Av. dos Bandeirantes, 3900 - 14040-902 - Ribeirão Preto, SP, Brasil

E-mail: layzebraz@usp.br 


\section{INTRODUÇÃO}

A tuberculose (TB) é considerada doença reemergente, devido ao aumento gradativo de casos nos últimos anos. Os principais fatores que predispõe à aquisição da TB seguem o modelo de triângulo epidemiológico de nexo e causalidade proposto por Gerstman, o qual expõe que a interação do bacilo, do homem e dos fatores ambientais aumentam a probabilidade de adquirir a TB ${ }^{(1)}$.

A despeito de parte das mortes causadas pela tuberculose serem preveníveis, esta se configura como a segunda maior causa de óbitos por doença infecciosa no mundo, perdendo apenas para o vírus da imunodeficiência humana (HIV). Em 2015, a Organização Mundial de Saúde (OMS) estimou que ocorreram 9,6 milhões de novos casos de TB em todo o mundo e cerca de um terço destes pacientes podem nunca ter sido diagnosticado ou tratado. Entre os novos casos, 1,2 milhão foi coinfectado pelo o vírus HIV e $0,4 \%$ das mortes ocorreram associadas à coinfecção TB-HIV(2).

A tuberculose, ocasionada pelo Mycobacterium tuberculosis (MTB), é uma infecção oportunista que acarreta exacerbação da carga viral e diminuição da contagem de CD4 em indivíduos com HIV. A infecção pelo HIV também pode alterar a patogênese da MTB levando a baciloscopia negativa, manifestações radiográficas atípicas e manifestações extrapulmonares, dificultando o diagnóstico desta doença ${ }^{(3)}$.

As pessoas que vivem com HIV são aproximadamente 30 vezes mais propensas a desenvolver TB quando comparadas às que não estão infectadas pelo vírus, de forma que a testagem para o HIV é recomendação padrão para todos os indivíduos com TB.

A cobertura do teste do HIV entre pacientes com tuberculose ainda é baixa nos serviços de saúde. No Brasil, a realização de testes rápidos do HIV para esses pacientes apresentou um bom desempenho em região do Centro-Oeste, entretanto conjectura-se que existam disparidades de desempenho entre as diferentes regiões do Brasil ${ }^{(4-5)}$.

A proposta do Programa Conjunto das Nações Unidas sobre HIV/AIDS (UNAIDS) era que 90\% das pessoas que possuíam o vírus do HIV conhecessem seu status sorológico até 2010. Apesar do teste para o HIV ter se tornado amplamente disponível, ainda existem barreiras na sua implementação. Países com alta epidemia como na África ainda subestimam a real magnitude dessa infecção. As principais barreiras que interferem nessa implementação são a ausência de orientação, baixa adesão por parte dos trabalhadores de saúde, a falta de kits do HIV e a supervisão inadequada por ambos os programas de TB e HIV ${ }^{(4,6-7)}$.

Diante disso, este estudo objetiva caracterizar o perfil epidemiológico da coinfecção TB/HIV, com o intuito de promover a discussão sobre assistência pelos serviços de saúde e proporcionar subsídios para implementar medidas de prevenção dessa coinfecção.

\section{- MÉTODO}

Trata-se de um estudo descritivo, epidemiológico, realizado por meio dos dados disponíveis no DATASUS, através do Sistema de Informação de Agravos de Notificação - SINAN no estado do Piauí. Os dados foram coletados em março de 2017 e são referentes aos anos de 2007 a 2016.

A coleta ocorreu por meio de instrumentos já existentes: as fichas de notificação do SINANNET nas quais está descrito o perfil epidemiológico da coinfecção TB/HIV. São fornecidas informações sobre o perfil sociodemográfico dos indivíduos, a situação de encerramento dos casos de TB e a realização de teste sorológico para detecção do HIV. A investigação foi realizada pelos próprios pesquisadores, que fazem parte da Gerência Estadual de Atenção à Saúde do Estado.

O SINAN é alimentado, principalmente, pela notificação e investigação de casos de doenças e agravos que constam da lista nacional de doenças de notificação compulsória ${ }^{(8)}$.

Após a coleta procedeu-se a tabulação dos dados no programa Excel. Realizou-se análise descritiva simples. A análise dos dados provenientes do SINAN foi realizada pelo programa TABNET. Os achados 
mais significativos foram apresentados em tabelas. A discussão dos dados foi feita com base na produção científica sobre a temática. O estudo foi submetido e aprovado pelo Comitê de Ética da Universidade Federal do Piauí, sob parecer número 1.576.964.

\section{RESULTADOS}

Dos 10.154 casos notificados de TB no estado do Piauí, entre os anos de 2007 a 2016, 619 (6,1\%) eram de coinfectados por HIV/TB. No ano de 2016, identificou-se o maior quantitativo de indivíduos diagnosticados com um total de 91 casos, e o menor quantitativo foi em 2009, com 35 casos, representando aumento heterogênio da prevalência dessa coinfecção (Gráfico 1).

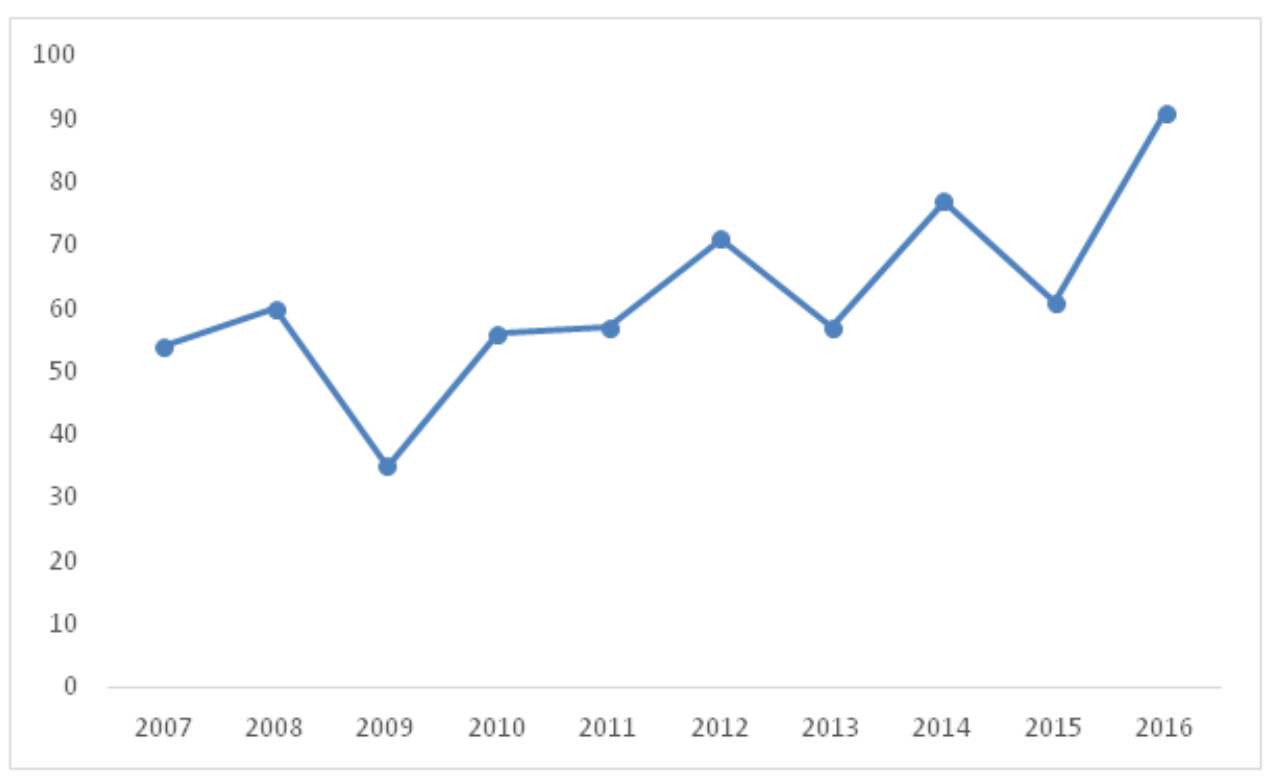

Gráfico 1 - Distribuição do HIV nos casos de Tuberculose, quanto ao ano no Piauí-2007 a 2016 (n=619). Teresina, PI, Brasil, 2017

Em relação ao perfil dos sujeitos, verificou-se predomínio de sexo masculino $(75,4 \%$; $n=467)$. As idades variaram entre menores 1 ano a maiores de 80 anos. Observou-se maior frequência de pessoas coinfectadas na faixa etária de 35-49 anos $(41,5 \%$; $n=257)$, seguido daqueles entre $20-34$ anos com 38\% $(n=235)$. Menores percentuais foram encontrados em crianças e adolescentes.

Tabela 1 - Distribuição do HIV nos casos de Tuberculose, quanto ao sexo, faixa etária e forma clínica no Piauí-2007 a 2016 ( $\mathrm{n=619)}$. Teresina, PI, Brasil, 2017 (continua)

\begin{tabular}{lcc} 
Variáveis & N & $\%$ \\
\hline Sexo & & \\
\hline Feminino & 152 & 25,6 \\
\hline Masculino & 467 & 75,4 \\
\hline Faixa etária (anos) & & \\
\hline$<1$ Ano & 2 & 0,3 \\
\hline $1-4$ & 6 & 1 \\
\hline $5-9$ & 3 & 0,5 \\
\hline $10-14$ & 5 & 0,8 \\
\hline
\end{tabular}




\begin{tabular}{lcc}
\hline $15-19$ & 6 & 1 \\
\hline $20-34$ & 235 & 38 \\
\hline $35-49$ & 257 & 41,5 \\
\hline $50-64$ & 89 & 14,3 \\
\hline $65-79$ & 13 & 2,1 \\
\hline $80 \mathrm{e}+$ & 3 & 0,5 \\
\hline Etnia & & \\
\hline Parda & 457 & 73,8 \\
\hline Branca & 60 & 9,7 \\
\hline Amarela & 5 & 0,8 \\
\hline Afrodescendente & 81 & 13,1 \\
\hline Ignorado/Branco & 16 & 2,6 \\
\hline Forma Cínica & & \\
\hline Pulmonar & 412 & 66,6 \\
\hline Extrapulmonar & 157 & 25.4 \\
\hline Pulmonar+Extrapulmonar & 50 & 8
\end{tabular}

Quanto à etnia, 73,8\% $(\mathrm{n}=457)$ dos indivíduos eram pardos, seguidos de brancos e afrodescendentes $9,7 \%(n=60)$ e $13,1 \%(n=81)$, respectivamente. Houve predominância da forma clínica do tipo pulmonar com $66,6 \%(n=412)$ das notificações, seguido pela TB extrapulmonar $25,4 \%(n=157)$ e $8 \%(n=50)$ dos casos apresentaram as duas formas da doença (pulmonar e extrapulmonar).

Tabela 2 - Situação de encerramento dos casos de Tuberculose no Estado do Piauí - 2007 a 2016 (n=10154). Teresina, PI, Brasil, 2017

\begin{tabular}{lcc} 
Situação de Encerramento & $\mathbf{N}$ & $\mathbf{\%}$ \\
\hline Cura & 6178 & 60,8 \\
\hline Abandono & 484 & 4,8 \\
\hline Óbito por Tuberculose & 351 & 3,5 \\
\hline Óbito por outras Causas & 385 & 3,8 \\
\hline Transferência & 2031 & 20 \\
\hline Tuberculose resistente a medicamentos (TB/DR) & 43 & 0,4 \\
\hline Mudança de Esquema & 6 & 0,06 \\
\hline Abandono Primário & 5 & 0,04 \\
\hline Ignorado/Branco & 671 & 6,6
\end{tabular}

Analisando os resultados da situação de encerramento dos casos de TB no estado, verificou-se índice de cura de $60,8 \%$ ( $n=6178$ ) e um número significativo de casos de abandono $(4,8 \% ; n=484)$, óbito $(3,5 \% ; n=351)$ e ignorado/branco $(6,6 \% ; n=671)$.

A Tabela 3 representa o percentual de pacientes com solicitação de teste sorológico para detecção do HIV, em relação ao total de casos de tuberculose, por ano de estudo. Nesta série histórica, o percentual de pacientes com tuberculose não testados para infecção pelo HIV diminuiu ao longo dos anos. De forma similar, o percentual de pacientes com resultado sorológico conhecido em relação ao número total de casos de tuberculose aumentou de 28,9\% em 2007 para 67,1\% em 2016 e, apenas 0,1\% $(\mathrm{n}=1)$ das notificações em 2015 foi branco/ignorado. 
Tabela 3 - Realização de teste sorológico para detecção do HIV, nos casos de tuberculose. 2007 a 2016 (n=10154). Teresina, PI, Brasil, 2017

\begin{tabular}{lccccccccc}
\multirow{2}{*}{$\begin{array}{l}\text { Ano } \\
\text { Diagnóstico }\end{array}$} & \multicolumn{2}{c}{ Positivo } & \multicolumn{2}{c}{ Negativo } & \multicolumn{2}{c}{ Em andamento } & \multicolumn{2}{c}{ Não realizado } & Total \\
\cline { 2 - 11 } & $\mathbf{n}$ & $\%$ & $\mathbf{n}$ & $\mathbf{\%}$ & $\mathbf{n}$ & $\mathbf{\%}$ & $\mathbf{n}$ & $\%$ & \\
\hline 2007 & 54 & 4 & 337 & 24,9 & 177 & 13 & 789 & 58,1 & 1357 \\
\hline 2008 & 60 & 4,9 & 373 & 30,5 & 256 & 20,9 & 535 & 43,7 & 1224 \\
\hline 2009 & 35 & 3,2 & 383 & 34,5 & 201 & 18,1 & 490 & 44,2 & 1109 \\
\hline 2010 & 56 & 5,6 & 478 & 48,1 & 42 & 4,2 & 418 & 42,1 & 994 \\
\hline 2011 & 57 & 5,7 & 451 & 45,1 & 56 & 5,6 & 436 & 43,6 & 1000 \\
\hline 2012 & 71 & 7,7 & 492 & 53,4 & 36 & 4,1 & 321 & 34,8 & 920 \\
\hline 2013 & 57 & 5,9 & 561 & 58,3 & 38 & 3,9 & 307 & 31,9 & 963 \\
\hline 2014 & 77 & 8,7 & 564 & 63,4 & 22 & 2,5 & 226 & 25,4 & 889 \\
\hline 2015 & 61 & 7,4 & 583 & 70,4 & 19 & 2,3 & 164 & 19,8 & 828 \\
\hline 2016 & 91 & 10,5 & 493 & 56,7 & 92 & 10,5 & 194 & 22,3 & 870 \\
\hline Total & 619 & 6,1 & 4715 & 46,4 & 939 & 9,2 & 3880 & 38,2 & 10154
\end{tabular}

As principais limitações do estudo envolvem a ausência do desfecho para os coinfectados TB/HIV, não apresentando desta forma a situação de encerramento dos casos. Ao longo dessa série histórica houve ascensão do número de coinfectados que cresceu de forma heterogênea (Gráfico 1), em contrapartida o numero de pacientes notificados com tuberculose sofreu um declínio com o passar dos anos (Tabela 3).

\section{- DISCUSSÃO}

A Organização Mundial de Saúde (OMS) apontou avanços no tratamento da tuberculose, com redução de 47\% na mortalidade entre 1990 e 2015. A incidência de TB diminuiu 1,4\% a cada ano desde 2000, entretanto o número de notificações aumentou para incidência de 10,4 milhões de casos em 2015, dos quais 11\% tinham infecção pelo HIV. As principais regiões que albergam essas duas infecções são África Subsaariana e os países da antiga União Soviética ${ }^{(9)}$.

A TB alterou as perspectivas de controle no mundo em virtude do aumento do número de casos. No Brasil, a região nordeste se apresenta como a mais endêmica para coinfecção TB/HIV, com taxas de incidência e de mortalidade que superam outras regiões como Centro-Oeste e Sul ${ }^{(10)}$.

O presente estudo, além de apontar o panorama da coinfecção TB-HIV, apresenta a abordagem ao identificar o diagnóstico do HIV em pacientes que inicialmente foram captados para o tratamento de $\mathrm{TB}$, por meio de triagem.

Quanto à caracterização sociodemográfica das pessoas com TB coinfectadas pelo HIV, destaca-se a predominância de indivíduos do sexo masculino, adultos com faixa etária entre 20 e 49 anos (79,5\%). Os resultados corroboram com estudo realizado em um país em desenvolvimento, o qual aponta que a grande maioria dessa coinfecção concentra-se no grupo etário economicamente produtivo (entre 25 a 49 anos). Este fato pode ser justificado devido a uma maior exposição às atividades realizadas nesta fase da vida, como relações sexuais, transfusões de sangue com seringas e agulhas contaminadas e uso de drogas injetáveis ${ }^{(11)}$.

Os fatores que determinam o adoecimento mais frequente em homens ainda não são claros, entretanto conjectura-se que possa estar relacionado com diversas condições, tanto de ordem biológica, relacionada ao autocuidado com a saúde, quanto ao baixo diagnóstico em mulheres ${ }^{(12)}$.

Referente à forma clínica, identificou-se o predomínio da tuberculose pulmonar, o que corrobora com outros estudos que abordam a coinfecção TB e HIV. Essa forma clínica é caracterizada por sua alta infectividade, desta forma, é premente a confirmação precoce da infecção por tuberculose, para que 
se possa interromper a cadeia de transmissão dessa doença ${ }^{(13-15)}$.

Um dos principais fatores que predispõe a alta prevalência dessa forma clínica é a predileção do patógeno pelo parênquima pulmonar. Deste modo, os problemas respiratórios estão entre as complicações mais frequentes em pessoas infectadas pelo HIV e a tuberculose torna-se a infecção oportunista mais comum e uma das principais causas de hospitalização nesse segmento populacional ${ }^{(16-17)}$.

O diagnóstico da tuberculose pulmonar em pacientes imunodeprimidos é caracterizado pela presença de tosse crônica de pelo menos 2 a 3 semanas, com febre vespertina, perda de peso, anorexia, astenia e sudorese noturna ${ }^{(17)}$.

No que diz respeito aos motivos de encerramento dos casos de TB, os percentuais de cura ainda estavam aquém dos valores pactuados pela OMS, que busca a cura em $85 \%$ dos casos novos. Inferese que o acompanhamento dos pacientes por meio das consultas favorece o sucesso do tratamento e consequente cura, especialmente por esclarecer dúvidas sobre a doença, o tratamento e a transmissão. A ausência do paciente nas consultas agendadas é indicativo de possível intenção de abandono do tratamento ${ }^{(18-19)}$.

Apesar do percentual de abandono do tratamento da TB se aproximar dos valores recomendados pela OMS $(5 \%)$, é pertinente salientar que essa problemática deve ser enfatizada, uma vez que se caracteriza como uma das principais limitações para a cura dessa infecção, além de proporcionar implicações para a sociedade como o aumento do desenvolvimento de tuberculose multirresistente, mortalidade e recidivas ${ }^{(16,18)}$.

A coinfecção TB-HIV é apontada como um fator que predispõe ao abandono do tratamento da tuberculose, visto que reações adversas e interações medicamentosas são mais intensas em pacientes soropositivos, fazendo com que muitas vezes o paciente opte pelo uso prioritário dos antirretrovirais em detrimento do medicamento para TB. Nessa perspectiva, foi proposta como meta a implementação do Tratamento Diretamente Observado (DOTS), com o intuito de minimizar ou até mesmo eliminar os casos de abandono do tratamento e estimular o uso correto do medicamento ${ }^{(18)}$.

Em relação à prevalência de pacientes coinfectados, os resultados encontrados permitem constatar que os achados nessa série histórica não refletem com exatidão a real magnitude dessa coinfecção, uma vez que mais da metade das fichas estavam preenchidas como "não realizado" ou "em andamento". A demora na liberação dos resultados e a ausência de atualização dos dados pelas Secretarias Municipais da Saúde podem favorecer esse alto percentual de preenchimento como "em andamento".

Infere-se que existam falhas estruturais nos serviços de saúde, tais como extravio de exames, demora no recebimento do resultado, dificuldade de acesso ao laboratório e até mesmo falta de atualização do sistema de informação. Nesta perspectiva, o diagnóstico precoce da coinfecção TB-HIV apresenta lacunas pela deficiência no fluxo de informação na rede assistencial e falta de infraestrutura adequada.

A coinfecção TB-HIV ainda se configura grande desafio para a saúde global, especialmente em países com recursos limitados e com grandes populações de indivíduos como a África e a Ásia, impondo desafios diagnóstico e terapêutico, exercendo enorme pressão sobre os sistemas de saúde(20).

A despeito da proposta do Plano Nacional de Controle da Tuberculose, que institui que todos os pacientes com tuberculose sejam submetidos ao teste anti-HIV, o que se constata é baixa frequência de solicitação e realização dessa sorologia. A principal implicação para essa prática é a incerteza sobre a real dimensão desta problemática ${ }^{(17,21)}$.

Diversas são as recomendações com o intuito de proporcionar subsídio ao controle da TB, como fortalecer a descentralização das ações de controle para Unidades de Saúde, e edificar uma rotina de busca, em outros sistemas de informação, para melhoria da qualidade de informação dos encerramentos dos casos de TB e avaliar o sistema de vigilância da tuberculose no município, a fim de definir as diretrizes e prioridades para melhorar os indicadores de cura, abandono e incidência da doença ${ }^{(22)}$.

Embora não faça parte do escopo desse estudo, é importante salientar que o principal desafio é a melhoria do SINAN-TB, tendo como meta principal a completude das informações, que por sua vez 
subsidiará a realização das análises dos agravos e possibilitará avaliação adequada, trazendo novos achados de valor epidemiológico.

O estudo apresentou como limitação o delineamento transversal que não possibilitou estabelecer relações de causa e o acompanhamento dos pacientes. Ademais, seus achados abrangem a caracterização epidemiológica da coinfecção tuberculose e o vírus da imunodeficiência de pacientes diagnosticados em um estado do nordeste do Brasil.

\section{CONCLUSÃO}

Neste estudo se observou que a maioria dos indivíduos coinfectados pela TB/HIV era do sexo masculino, na faixa etária de 35 a 49 anos e pardos. A tuberculose pulmonar foi a forma mais prevalente. Houve distribuição heterogênea dos coinfectados ao longo do período de estudo.

O desfecho do tratamento da tuberculose caracteriza-se de forma majoritária com a cura. Entretanto, apesar do acesso ao tratamento dessas duas infecções e a ampliação da prática de testagem rápida para o HIV, urge a implementação de intervenções adequadas às múltiplas circunstâncias, com o intuito de alcançar níveis elevados de cura dos pacientes acometidos por TB e detecção dos coinfectados por TB e HIV.

Embora não seja recomendada a detecção do HIV a partir dos casos de infecção por tuberculose, observa-se que essa realidade se faz presente. Nesse contexto, é pertinente a identificação precoce dessa infecção, assim como a implementação de testes rápidos para detectar os casos de HIV antes da instalação de doenças oportunistas e da AIDS.

Apesar dos esforços empreendidos pelas equipes de saúde, a coinfecção ainda representa grave problema, visto que pessoas contaminadas por ambas as infecções são potentes fontes de disseminação da doença. Nessa perspectiva, é importante que o Estado leve em consideração a realidade local e busque medidas para a diminuição e combate a essas doenças.

\section{REFERÊNCIAS}

1. Khan AH. Tuberculosis control in Sindh, Pakistan: Critical analysis of its implementation. J Infec Public Health. [Internet] 2017;10(1) [acesso em 19 jul 2016]. Disponível: http://dx.doi.org/10.1016/j.jiph.2016.02.007.

2. World Health Organization (WHO). Global Tuberculosis Report - 2015. [Internet] Geneva: WHO; 2015 [acesso em 19 jul 2016]. Disponível: http://apps.who.int/iris/bitstream/10665/191102/1/9789241565059_eng.pdf?ua=1.

3. Montales MT, Beebe A, Chaudhury A, Patil N. Mycobacterium tuberculosis infection in a HIV-positive patient. Respir Med Case Rep. [Internet] 2015;(16) [acesso em 19 jul 2016]. Disponível: http://dx.doi.org/10.1016/j. rmcr.2015.10.006.

4. Trinh QM, Nguyen HL, Nguyen VN, Nguyen TV, Sintchenko V, Marais BJ. Tuberculosis and HIV co-infectionfocus on the Asia-Pacific region. Int J Infect Dis. [Internet] 2015;(32) [acesso em 19 jul 2016]. Disponível: http:// dx.doi.org/10.1016/j.ijid.2014.11.023.

5. Bartholomay P, Pelissari DM, de Araujo WN, Yadon ZE, Heldal E. Quality of tuberculosis care at different levels of health care in Brazil in 2013. Rev Panam Salud Publica. [Internet] 2016;39(1) [acesso em 30 out 2017]. Disponível: http://www.scielosp.org/pdf/rpsp/v39n1/1020-4989-RPSP-39-01-003.pdf.

6. United Nations Programme on HIV/AIDS (UNAIDS). The Gap Report. [Internet] 2014 [acesso em 30 out 2017]. Disponível: http://www.unaids.org/sites/default/files/media_asset/UNAIDS_Gap_report_en.pdf.

7. Velen K, Lewis JJ, Charalambous S, Page-Shipp L, Popane F, Churchyard GJ, et al. Household HIV testing uptake among contacts of TB patients in South Africa. PLoS One. [Internet] 2016;11(5) [acesso em 30 out 2017]. Disponível: http://dx.doi.org/10.1371/journal.pone.0155688.

8. Ministério da Saúde (BR). Secretaria de Vigilância em Saúde. Departamento de Vigilância Epidemiológica. 
Sistema de Informação de Agravos de Notificação - Sinan: normas e rotinas. Brasília: Ministério da Saúde; 2007.

9. World Health Organization (WHO). Global tuberculosis report 2016. WHO library cataloguing-in-publication data. [Internet] Geneva: WHO; 2016 [acesso em 20 out 2017]. Disponível: http://apps.who.int/iris/bitstre am/10665/250441/1/9789241565394-eng.pdf.

10. Barbosa IR, Costa ICC. Estudo epidemiológico da coinfecção tuberculose-hiv no nordeste do Brasil. Rev Patol Trop. [Internet] 2014;43(1) [acesso em 20 jul 2016]. Disponível: https://doi.org/10.5216/rpt.v43i1.29369.

11. Ranti KO, Glory AO, Victoria BT, Isaac KO. Prevalence of HIV infection among tuberculosis patients in a teaching hospital in south-west Nigeria: A four-year retrospective study. HIV \& AIDS Review [Internet] 2016;15(4) [acesso em 22 jul 2016]. Disponível: https://doi.org/10.1016/j.hivar.2016.11.001.

12. Hino P, Takahashi RF, Bertolozzi MR, Egry EY. Coinfecção de Tb/HIV em um distrito administrativo do Município de São Paulo. Acta paul. enferm. [Internet] 2012;25(5) [acesso em 22 jul 2016]. Disponível: http://dx.doi. org/10.1590/S0103-21002012000500017.

13. Tiberi S, Carvalho ACC, Sulis G, Vaghela D, Rendon A, Mello FCQ, et al. The cursed duet today: Tuberculosis and HIV-coinfection. Presse Med. [Internet] 2017;46(2 pt 2) [acesso em 19 jul 2016]. Disponível: http://dx.doi. org/10.1016/j.lpm.2017.01.017.

14. Kumar AMV, Singarajipura A, Naik B, Guddemane DK, Patel Y, Shastri S, et al. HIV-infected presumptive tuberculosis patients without tuberculosis: How many are eligible for antiretroviral therapy in Karnataka, India? J Epidemiol Glob Health. [Internet] 2017;7(1) [acesso em 19 jul 2016]. Disponível: http://dx.doi.org/10.1016/j. jegh.2015.12.002.

15. Rivest P, Sinyavskaya L, Brassard P. Burden of HIV and tuberculosis co-infection in Montreal, Quebec. Can J Public Health. [Internet] 2014;105(4) [acesso em 19 jul 2016]. Disponível: https://journal.cpha.ca/index.php/cjph/ article/viewFile/4269/2939.

16. da Silva EG, Vieira JDS, Cavalcante AL, Santos LGML, Rodrigues APRA, Cavalcante TCS. Perfil epidemiológico da tuberculose no estado de alagoas de 2007 a 2012. Ciências Biológicas e da Saúde. [Internet] 2015;3(1) [acesso em 19 jul 2016]. Disponível: https://periodicos.set.edu.br/index.php/fitsbiosaude/article/view/2352.

17. Horo K, Koné A, Koffi MO, Ahui JMB, Brou-Godé CV, Kouassi AB, et al. Diagnostic comparé des pneumopathies bactériennes et de la tuberculose pulmonaire chez les patients VIH+. Rev Mal Respir. [Internet] 2016;33(1) [acesso em 19 jul 2016]. Disponível: https://doi.org/10.1016/j.rmr.2015.01.004.

18. Ministério da Saúde (BR). Secretaria de Vigilância em Saúde. Departamento de Vigilância Epidemiológica. Manual para recomendações para o controle da tuberculose no Brasil. Brasília: Ministério da Saúde; 2011.

19. de Lima LM , Harter J, Tomberg JO, Vieira DA, Antunes ML, Cardozo-Gonzales RI. Avaliação do acompanhamento e desfecho de casos de tuberculose em município do sul do Brasil. Rev. Gaúcha Enferm. [Internet] 2016;37(1) [acesso em 18 jul 2016]. Disponível: http://dx.doi.org/10.1590/1983-1447.2016.01.51467.

20. Pawlowski A, Jansson M, Sköld M, Rottenberg ME, Källenius G. Tuberculosis and HIV Co-Infection. PLoS Pathog. [Internet] 2012;8(2) [acesso em 19 jul 2016]. Disponível: http://dx.doi.org/10.1371/journal.ppat.1002464.

21. Siqueira KZ, Mendonça SA, Penedo CC. Indicação da prova tuberculínica e infecção latente da tuberculose em HIV-positivos, Município de Blumenau, Estado de Santa Catarina, Brasil, 2004-2009. Epidemiol. Serv. Saúde. [Internet] 2012;21(4) [acesso em 19 jul 2016]. Disponível: http://dx.doi.org/10.5123/S1679-49742012000400013.

22. Pereira JC, Silva MR, da Costa RR, Guimarães MDC, Leite ICG. Perfil e seguimento dos pacientes com tuberculose em município prioritário no Brasil. Rev. Saúde Pública. [Internet] 2015;(49) [acesso em 19 jul 2016]. Disponível: http://dx.doi.org/10.1590/S0034-8910.2015049005304. 